\title{
Numerical study for fractional model of nonlinear predator-prey biological population dynamic system
}

\author{
Jagdev Singh ${ }^{1}$, A. Kilicman*2, Devendra Kumar ${ }^{3}$ and Ram Swroop ${ }^{4}$ \\ ${ }^{1}$ Department of Mathematics, JECRC University, Jaipur-303905, Rajasthan, India \\ ${ }^{2}$ Department of Mathematics and Institute for Mathematical Research University Putra Malaysia, \\ 43400 UPM, Serdang, Selangor, Malaysia \\ ${ }^{3}$ Department of Mathematics, University of Rajasthan, Jaipur-302004, Rajasthan, India \\ ${ }^{4}$ Department of Mathematics, Arya Institute of Engineering \& Technology, Riico Kukas, Jaipur- \\ 303101, Rajasthan, India \\ Abstract. The key objective of the present paper is to propose a numerical scheme based on the \\ homotopy analysis transform technique to analyze the time-fractional nonlinear predator-prey \\ population model. The population model is coupled fractional order nonlinear partial differential \\ equations often employed to narrate the dynamics of biological systems in which two species \\ interact, first is a predator and the second is a prey. The proposed scheme provides the series \\ solution with great freedom and flexibility by choosing appropriate parameters. The convergence \\ of results is free from small or large parameters. Three examples are discussed to demonstrate \\ the correctness and efficiency of the used computational approach.
}

Key words: Fractional nonlinear predator-prey population model; Biological systems; Laplace transform algorithm; Homotopy analysis transform technique

2010 Mathematics Subject Classification: 34A08, 35A20, 35A22.

\footnotetext{
* Corresponding author: Tel.

Email address: jagdevsinghrathore@gmail.com (J. Singh), akilicman@putra.upm.edu.my (A.

Kilicman*),devendra.maths@gmail.com (D. Kumar), mathsram09@gmail.com (R. Swroop).
} 


\section{Introduction}

The fractional calculus concerns with the integral and derivative operators of arbitrary order. The history of fractional calculus is nearly same as the differential calculus; its period can be stretched from $16^{\text {th }}$ century when the derivative of order $\alpha=1 / 2$ was explained by Leibnitz [1]. There are many monographs [2-3] that contains a detail account of fractional calculus and its various related issues. Fractional calculus is an aid for describing natural phenomena of nonlocal nature. The fractional model of population growth is formulated in [4] which is very useful in abnormal situations.

In nowadays, ample interest in differential equations of fractional order has been triggered due to their large number of utilities in modeling of many engineering and scientific processes [511]. In comparison of the standard differential equations, in which derivatives depend only on the local nature of the function, differential equations having fractional order assemble the complete information of the function in a weighted form. This is well known as memory effect. The examination of fractional generalization of differential equation is much completed.

In this work, we put up the combined form of homotopy analysis method (HAM) and classical Laplace transform to produce a modified algorithm termed as the homotopy analysis transform method (HATM) [12-15] to analyze the time-fractional nonlinear predator-prey population model. HAM was advised by Liao [16-20] a researcher of Shanghi Jiao Tong University in his PhD thesis in 1992. A monograph by Liao systematically presents the fundamental theory of the HAM, its connection with other analytic approaches, and some of its importance in scientific and engineering areas [21]. 
The dynamical connection between predator and prey is the main issue in ecology and mathematical sciences. The considerable development in population dynamic was presented independently by many research workers [22-23]. They formulated firstly the mathematical model of predator-prey type. In an attempt author of [24] formulated a mathematical equation namely logistic equation by using principle of population. First of all a systematic explanation of population dynamics was given by the author of [25] in his report. In a work author of [26] investigated a predator-prey model along with Michaelis-Menten type. Much more remarkable research work was conducted by many scientists and they improved and modified these types of mathematical models by using different methodologies [27-34]. In a recent work the fractional model of predator-prey system is solved by using homotopy perturbation technique [35].

We appraise the two-species competitive model by taking into consideration population of prey and population of predator $v$. For population of prey $u \rightarrow 2 u$, having the rate $\lambda, \lambda>0$ indicates the rate of natural birth. For population of predator $v \rightarrow 0$, having the rate $\mu, \mu>0$ stands for the rate of natural death. The connected term between predator and prey populations is $u+v \rightarrow$ $2 v$, at rate $\sigma$, parameter $\sigma$ designate the competitive rate. In view of an extensively accepted theory of fractional biological population models, the mathematical model of a predator-prey system of fractional order can be illustrated as

$$
\begin{aligned}
& \frac{\partial^{\rho} \xi}{\partial t^{\rho}}=\frac{\partial^{2} \xi}{\partial x^{2}}+\frac{\partial^{2} \xi}{\partial y^{2}}+\lambda \xi-\sigma \xi \eta, \quad \xi(x, y, 0)=\varphi(x, y), \\
& \frac{\partial^{\beta} \eta}{\partial t^{\beta}}=\frac{\partial^{2} \eta}{\partial x^{2}}+\frac{\partial^{2} \eta}{\partial y^{2}}+\sigma \xi \eta-\mu \eta, \quad \eta(x, y, 0)=\phi(x, y) .
\end{aligned}
$$

In the above Eq. (1) $t>0, x, y \in R, \lambda, \sigma, \mu>0$, the function $\xi$ represents the density of prey population and the function $\eta$ indicates the density of predator population, $\varphi(x, y), \phi(x, y)$ indicates initial conditions for the growth of population. 
The objective of this article is to enhance the utilization of homotopy analysis transform technique (HATT) to investigate the nonlinear predator-prey biological population dynamical system of fractional order. The advantage of the suggested computational scheme is that it provides a system of creating a set of base functions and yields the high order deformation equations in simpler, linear sub problems by choosing appropriate initial approximations.

\section{Basic definitions}

Definition 1. The Riemann-Liouville (RL) integral of arbitrary order $\rho>0$, of a function $\xi(t) \in C_{\mu}, \mu \geq-1$ is given as follows

$J^{\rho} \xi(t)=\frac{1}{\Gamma(\alpha)} \int_{0}^{t}(t-z)^{\rho-1} \xi(z) d z,(\rho>0)$

$J^{0} \xi(t)=\xi(t)$

for the RL fractional integral, the following results hold

(i) $J^{\rho_{1}} J^{\rho_{2}} \xi(t)=J^{\rho_{1}+\rho_{2}} \xi(t)$,

(ii) $J^{\rho_{1}} J^{\rho_{2}} \xi(t)=J^{\rho_{2}} J^{\rho_{1}} \xi(t)$,

(iii) $J^{\rho} t^{\delta}=\frac{\Gamma(\delta+1)}{\Gamma(\rho+\delta+1)}$.

Definition 2. The derivative of fractional order of $\xi(t)$ proposed by Caputo is given as [36]:

$D^{\rho} \xi(t)=J^{n-\rho} D^{n} \xi(t)$

$$
=\frac{1}{\Gamma(n-\alpha)} \int_{0}^{t}(t-z)^{n-\rho-1} \xi^{n}(z) d z
$$

for $n-1<\rho \leq n, n \in N, t>0, \xi \in C_{-1}{ }^{n}$,

Lemma: If $n-1<\rho \leq n, n \in N, \xi \in C_{\mu}{ }^{n}, \mu \geq-1$, then the following result holds 
$D^{\rho} J^{\rho} \xi(t)=\xi(t)-\sum_{r=0}^{n-1} \xi^{(r)}\left(0^{+}\right) \frac{t^{r}}{\Gamma(r+1)}, t>0$.

In the present work we employ the fractional derivative termed in Caputo due to its ability to permit the traditional initial and boundary conditions involved in the mathematical modelling.

Definition 3. For the smallest integer $\mathrm{r}$ that exceeds $\rho$, the derivative of arbitrary order introduced by Caputo of order $\rho>0$ is given as follows

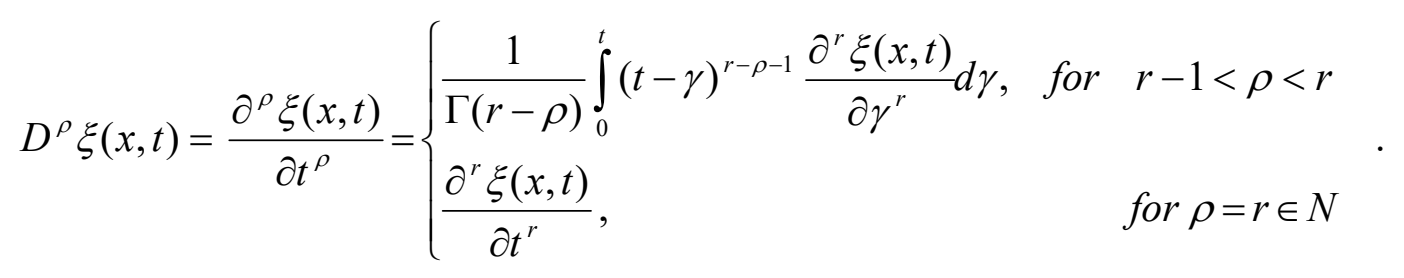

Definition 4. Suppose $\xi(t)$ be function of $t$ then the Laplace transform (LT) of $\xi(t)$ is expressed by

$L\{\xi(t), s\}=\bar{\xi}(s)=\int_{0}^{\infty} e^{-s t} \xi(t) d t$

If $n \in N$, then LT is given as

$L\left\{\frac{d^{n} \xi}{d x^{n}} ; s\right\}=s^{n} \bar{\xi}(s)-\sum_{r=0}^{n-1} s^{n-r-1} \xi^{(r)}\left(0^{+}\right)$,

or the LT of Caputo derivative [36,37] is expressed in the form

$L\left[D^{\rho} \xi(t)\right]=s^{\rho} L[\xi(t)]-\sum_{r=0}^{n-1} s^{\rho-r-1} \xi^{(r)}\left(0^{+}\right), n-1<\rho \leq n$.

\section{Basic concept of homotopy analysis transform technique}

We assume a nonlinear PDE involving fractional derivative of the form:

$D_{t}^{\rho} \xi+R \xi+N \xi=a(t), \quad n-1<\rho \leq n$. 
In Eq. (11) $D_{t}^{\rho} \xi$ is representing the fractional derivative of $\xi$ understood in Caputo sense, $R$ is standing for the linear differential operator, $\mathrm{N}$ is indicating the general nonlinear differential operator and $a(t)$ is representing the term due to source.

By putting the LT operator on Eq. (11), we get

$L\left[D_{t}^{\rho} \xi\right]+L[R \xi]+L[N \xi]=L[a(t)]$

Employing the fractional differentiation formula the LT, we obtain

$s^{\rho} L[\xi]-\sum_{k=0}^{n-1} s^{\alpha-k-1} \xi^{(k)}(0)+L[R \xi]+L[N \xi]=L[a(t)]$.

If we simplify the Eq. (13), it gives

$L[\xi]-\frac{1}{s^{\rho}} \sum_{k=0}^{n-1} s^{\rho-k-1} \xi^{(k)}(0)+\frac{1}{s^{\rho}}[L[R \xi]+L[N \xi]-L[a(t)]]=0$.

We interpret the nonlinear operator

$$
\begin{array}{r}
N[\vartheta(t ; z)]=L[\vartheta(t ; z)]-\frac{1}{s^{\rho}} \sum_{k=0}^{n-1} s^{\rho-k-1} \vartheta^{(k)}(t ; z)\left(0^{+}\right) \\
+\frac{1}{s^{\rho}}[L[R \vartheta(t ; z)]+L[N \vartheta(t ; z)]-L[a(t)]]
\end{array}
$$

where $z \in[0,1]$ and $\mathcal{Y}(t ; z)$ is a real function of $\mathrm{t}$ and $\mathrm{z}$. We develop a homotopy as

$$
(1-z) L\left[\vartheta(t ; z)-\xi_{0}(t)\right]=\hbar z N[\xi(t)]
$$

In the expression (16) $\mathrm{L}$ is indicating the $\mathrm{LT}$ operator, $z \in[0,1]$ is denoting the embedding parameter, $\hbar \neq 0$ is standing for an auxiliary parameter, $\xi_{0}$ is denoting an initial guess of $\xi$ and $\vartheta(t ; z)$ is indicating an unknown function. Clearly, when the embedding parameter $z=0$ and $z=1$, it yields

$\vartheta(t ; 0)=\xi_{0}(t), \quad \vartheta(t ; 1)=\xi(t)$, 
respectively. It is to be noticed that as $z$ increases form 0 to 1 , the solution $\vartheta(t ; z)$ converts from the initial guess $\xi_{0}$ to the solution $\xi$. Now using the Taylor theorem we write the $\vartheta(t ; z)$ in series form given below

$\vartheta(t ; z)=\xi_{0}(t)+\sum_{m=1}^{\infty} \xi_{m}(t) z^{m}$

where

$\xi_{m}(t)=\left.\frac{1}{m !} \frac{\partial^{m} \vartheta(t ; q)}{\partial z^{m}}\right|_{z=0}$

If the initial approximation of the solution and the parameter $\hbar$ are taken in well manner, the series (18) converges at $z=1$, then we have the subsequent series solution of the given nonlinear equation of fractional order

$\xi=\xi_{0}+\sum_{m=1}^{\infty} \xi_{m}$

Making use of (20), the governing equation can be derived from the Eq. (16).

Now we define the following vectors

$\bar{\xi}_{m}=\left\{\xi_{0}, \xi_{1}, \ldots, \xi_{m}\right\}$.

On differentiation of the Eq. (16) m-times w.r.t. $z$ and then division them by $m$ ! and then putting $z=0$, we arrive at the following result:

$L\left[\xi_{m}-\chi_{m} \xi_{m-1}\right]=\hbar \Re_{m}\left(\bar{\xi}_{m-1}\right)$

Applying the inverse LT, we get the following result

$\xi_{m}=\chi_{m} \xi_{m-1}+\hbar L^{-1}\left[\Re_{m}\left(\bar{\xi}_{m-1}\right)\right]$

where 
$\mathfrak{R}_{m}\left(\bar{\xi}_{m-1}\right)=\left.\frac{1}{(m-1) !} \frac{\partial^{m-1} N[\vartheta(t ; z)]}{\partial z^{m-1}}\right|_{z=0}$,

and

$\chi_{m}= \begin{cases}0, & m \leq 1 \\ 1, & m>1\end{cases}$

\section{Implementation of the method}

Here we apply the HATT to examine the fractional predator-prey system given in Eq. (1). We express the nonlinear operator as

$$
\begin{aligned}
& N_{1}\left[\vartheta_{1}(x, y, t ; z), \vartheta_{2}(x, y, t ; z)\right] \\
& =L\left[\vartheta_{1}(x, y, t ; z)\right]-\left(1-\chi_{m}\right) \frac{1}{s} \varphi(x, y)-\frac{1}{s^{\rho}} L\left[\frac{\partial^{2} \vartheta_{1}}{\partial x^{2}}+\frac{\partial^{2} \vartheta_{1}}{\partial y^{2}}+\lambda \vartheta_{1}-\sigma \vartheta_{1} \vartheta_{2}\right]
\end{aligned}
$$

and

$$
\begin{aligned}
& N_{2}\left[\vartheta_{1}(x, y, t ; z), \vartheta_{2}(x, y, t ; z)\right] \\
& =L\left[\vartheta_{2}(x, y, t ; z)\right]-\left(1-\chi_{m}\right) \frac{1}{s} \phi(x, y)-\frac{1}{s^{\beta}} L\left[\frac{\partial^{2} \vartheta_{2}}{\partial x^{2}}+\frac{\partial^{2} \vartheta_{2}}{\partial y^{2}}+\sigma \vartheta_{1} \vartheta_{2}-\mu \vartheta_{2}\right]
\end{aligned}
$$

Further the LT operators are written as

$$
L\left[\xi_{m}(x, y, t)-\chi_{m} \xi_{m-1}(x, y, t)\right]=\hbar R_{1, m}\left[\vec{\xi}_{m-1}, \vec{\eta}_{m-1}\right]
$$

and

$$
L\left[\eta_{m}(x, y, t)-\chi_{m} \eta_{m-1}(x, y, t)\right]=\hbar R_{2, m}\left[\vec{\xi}_{m-1}, \vec{\eta}_{m-1}\right]
$$

In the above Eqs. (28) and (29), the $R_{1, m}\left[\vec{\xi}_{m-1}, \vec{\eta}_{m-1}\right]$ and $R_{2, m}\left[\vec{\xi}_{m-1}, \vec{\eta}_{m-1}\right]$ are expressed as

$$
\begin{aligned}
R_{1, m}\left[\vec{\xi}_{m-1}, \vec{\eta}_{m-1}\right]= & L\left\{\xi_{m-1}(x, y, t)\right\}-\left(1-\chi_{m}\right) \frac{1}{s} \xi_{0} \\
& -\frac{1}{s^{\alpha}} L\left\{\frac{\partial^{2} \xi_{m-1}}{\partial x^{2}}+\frac{\partial^{2} \xi_{m-1}}{\partial y^{2}}+\lambda \xi_{m-1}-\sigma \sum_{i=0}^{m-1} \xi_{i} \eta_{m-1-i}\right\}
\end{aligned}
$$


and

$$
\begin{aligned}
R_{2, m}\left[\vec{\xi}_{m-1}, \vec{\eta}_{m-1}\right]= & L\left\{\eta_{m-1}(x, y, t)\right\}-\left(1-\chi_{m}\right) \frac{1}{s} \eta_{0} \\
& -\frac{1}{s^{\beta}} L\left\{\frac{\partial^{2} \eta_{m-1}}{\partial x^{2}}+\frac{\partial^{2} \eta_{m-1}}{\partial y^{2}}+\sigma \sum_{i=0}^{m-1} \xi_{i} \eta_{m-1-i}-\mu \eta_{m-1}\right\}
\end{aligned}
$$

It is obvious that the solution of Eqs. (28) and (29) for, $\mathrm{m} \geq 1$ becomes

$$
\xi_{m}=\chi_{m} \xi_{m-1}+\hbar L^{-1}\left\{R_{1, m}\left[\vec{\xi}_{m-1}, \vec{\eta}_{m-1}\right]\right\}
$$

and

$$
\eta_{m}=\chi_{m} \eta_{m-1}+\hbar L^{-1}\left\{R_{2, m}\left[\vec{\xi}_{m-1}, \vec{\eta}_{m-1}\right]\right\}
$$

The approximate solutions of the original Eq. (1) can be expressed as

$$
\xi=\sum_{r=0}^{\infty} \xi_{r}
$$

and

$$
\eta=\sum_{r=0}^{\infty} \eta_{r}
$$

\section{Numerical simulation for fractional model of Predator-Prey system}

In order to show and effectiveness and correctness of the HATT for analyzing the fractional model of nonlinear partial differential equation, we apply it to the subsequent several initial conditions problems.

Case 1. Firstly, we take the predator-prey system of fractional order along with the constant initial conditions

$$
\xi(x, y, 0)=\xi_{0}, \quad \eta(x, y, 0)=\eta_{0} .
$$

By appealing to the HATT to solve the Eqs. (32) and (33) with initial condition (36) we get the following results 


$$
\begin{aligned}
& \xi(x, y, 0)=\xi_{0} \quad \eta(x, y, 0)=\eta_{0}, \\
& \xi_{1}=-\frac{\hbar\left(\lambda \xi_{0}-\sigma \xi_{0} \eta_{0}\right) t^{\rho}}{\Gamma(\rho+1)}, \quad \eta_{1}=-\frac{\hbar\left(\sigma \xi_{0} \eta_{0}-\mu \eta_{0}\right) t^{\beta}}{\Gamma(\beta+1)}, \\
& \xi_{2}=-\frac{\hbar(\hbar+1)\left(\lambda \xi_{0}-\sigma \xi_{0} \eta_{0}\right) t^{\rho}}{\Gamma(\rho+1)}+\frac{\hbar^{2} \xi_{0}\left(\lambda-\sigma \eta_{0}\right)^{2} t^{2 \rho}}{\Gamma(2 \rho+1)}+\frac{b \hbar^{2} \xi_{0} \eta_{0}\left(\mu-\sigma \xi_{0}\right) t^{\rho+\beta}}{\Gamma(\rho+\beta+1)}, \\
& \eta_{2}=-\frac{\hbar(\hbar+1)\left(\sigma \xi_{0} \eta_{0}-\mu \eta_{0}\right) t^{\beta}}{\Gamma(\beta+1)}+\frac{\hbar^{2} \eta_{0}\left(\mu-\sigma \xi_{0}\right)^{2} t^{2 \beta}}{\Gamma(2 \beta+1)}+\frac{\sigma \hbar^{2} v_{0}\left(\lambda \xi_{0}-\sigma \xi_{0} \eta_{0}\right) t^{\rho+\beta}}{\Gamma(\rho+\beta+1)},
\end{aligned}
$$

In the same way the remaining terms can be obtained. The HATT solutions of the original Eq. (1) with the initial condition (36) obtained in series form as

$$
\xi=\xi_{0}+\xi_{1}+\xi_{2}+\cdots, \quad \eta=\eta_{0}+\eta_{1}+\eta_{2}+\cdots
$$

It's clear to see the Figs. 1-4, initially prey population density increased up and after that it lower down with the increase in time t. Although in the same way predator population density always increases with the special variables with parameters we select in this problem. The numerical results found by the HATT are very near to the anomalous biological diffusion nature seen in the real world, with high accuracy at the third-term approximations. 


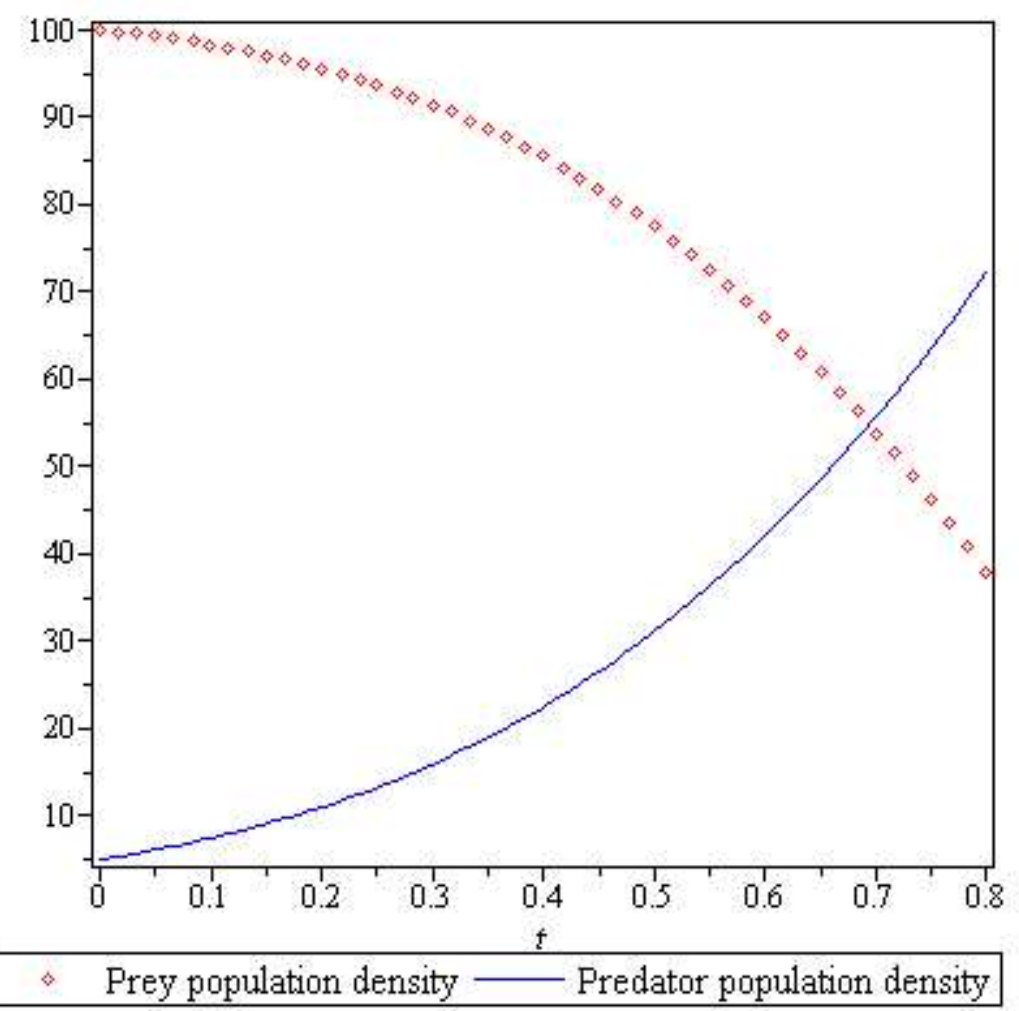

Figure 1. Time evaluation of prey $\xi$ and predator $\eta$ population density for (38) when $\xi_{0}=100, \eta_{0}=5, \lambda=0.08, \sigma=0.04, \mu=0.02$ and $\hbar=-1$, with $\rho=\beta=1$. 


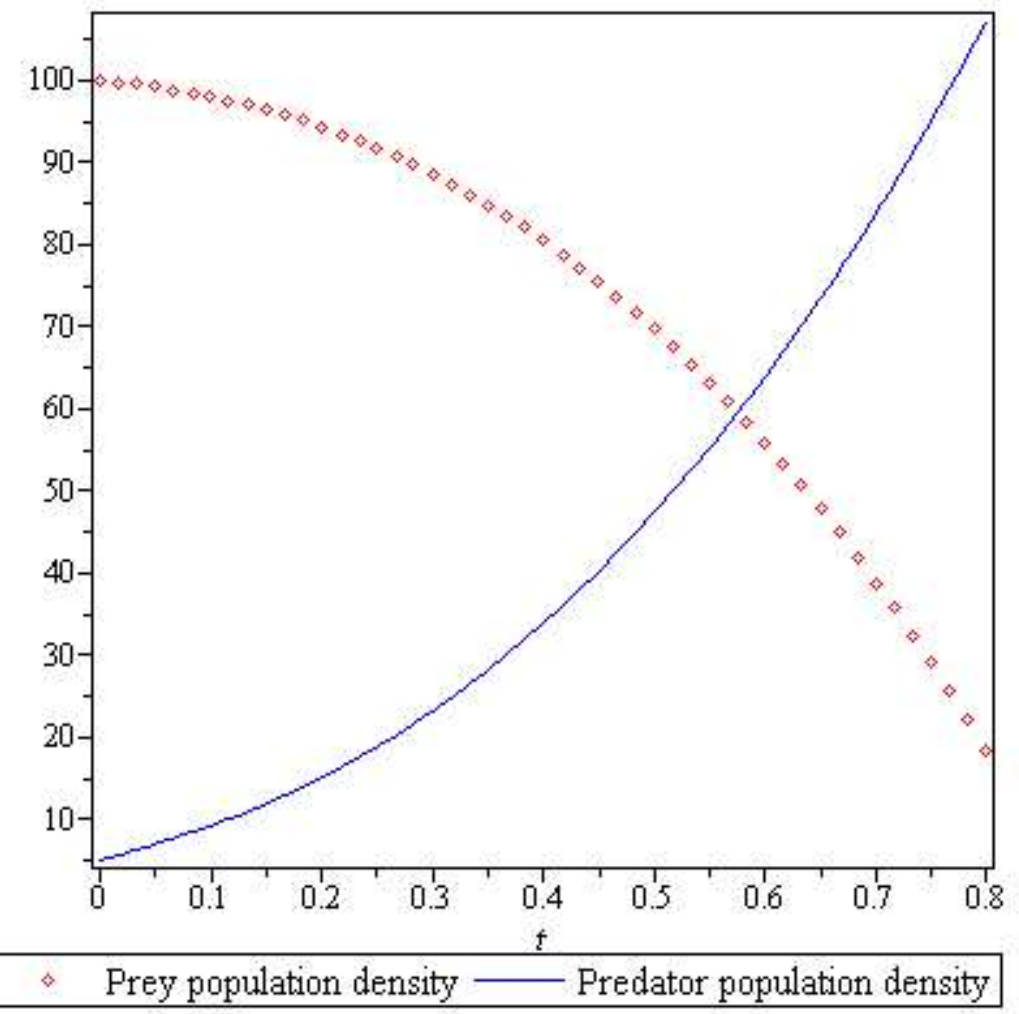

Figure 2. Time evaluation of prey $\xi$ and predator $\eta$ population density for (38) when $\xi_{0}=100, \eta_{0}=5, \lambda=0.08, \sigma=0.04, \mu=0.02$ and $\hbar=-1$, with $\rho=1$ and $\beta=0.85$. 


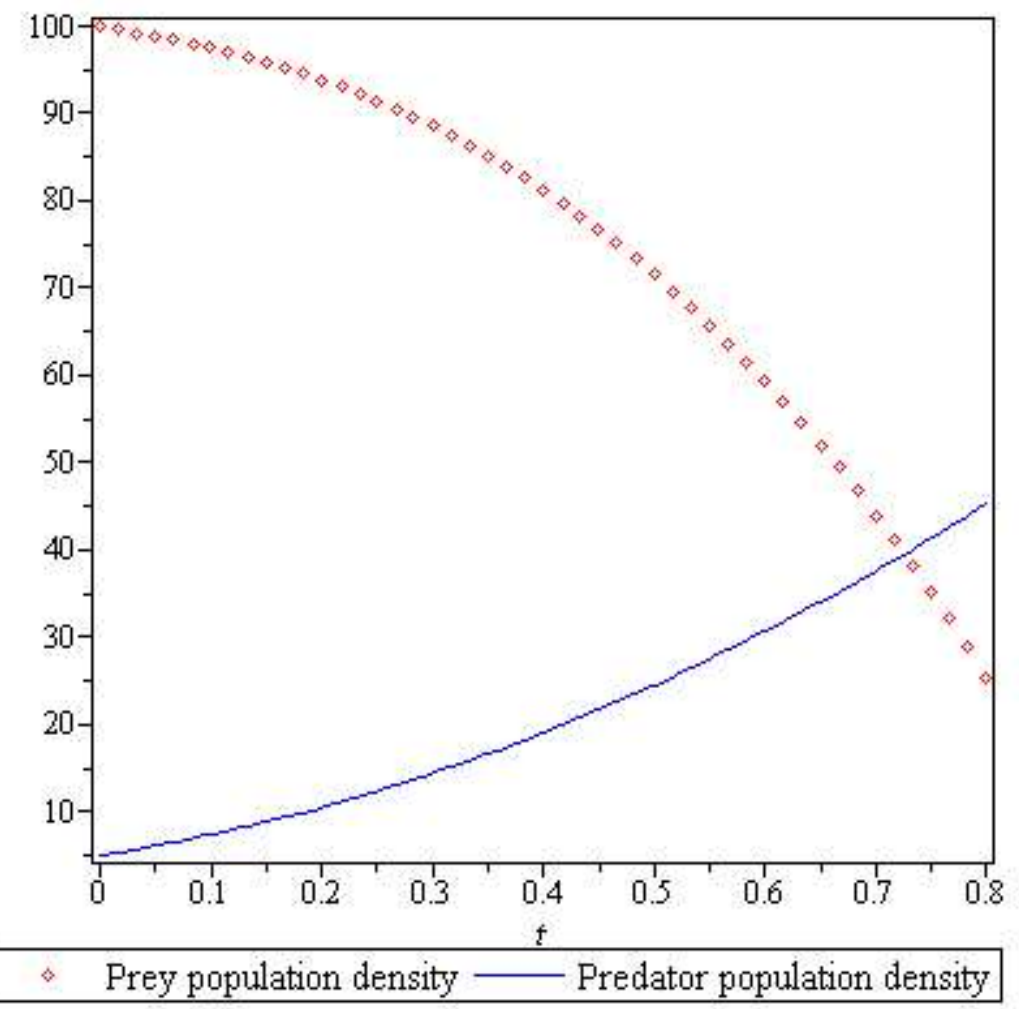

Figure 3. Time evaluation of prey $\xi$ and predator $\eta$ population density for (38) when $\xi_{0}=100, \eta_{0}=5, \lambda=0.08, \sigma=0.04, \mu=0.02$ and $\hbar=-1$, with $\rho=0.85$ and $\beta=1$. 


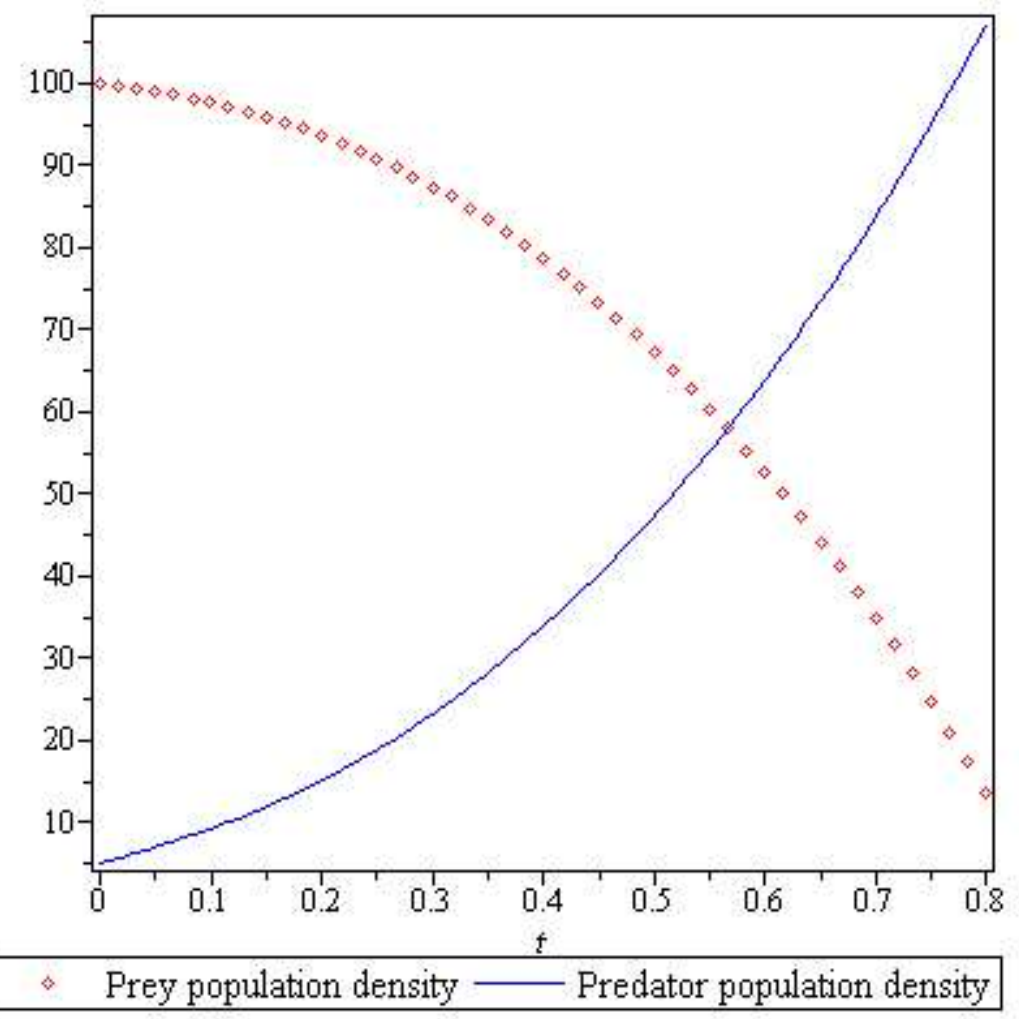

Figure 4. Time evaluation of prey $\xi$ and predator $\eta$ population density for (38) when $\xi_{0}=100, \eta_{0}=5, \lambda=0.08, \sigma=0.04, \mu=0.02$ and $\hbar=-1$, with $\rho=0.95$ and $\beta=0.60$.

Case 2. Secondly, we assume the predator-prey equation of fractional order having the initial conditions

$\xi(x, y, 0)=e^{x+y}, \quad \eta(x, y, 0)=e^{x+y}$.

By using the HATT to solve the Eqs. (32) \& (33) with initial conditions (39), we get

$\xi_{0}=e^{x+y}$ $\eta_{0}=e^{x+y}$ $\xi_{1}=-\frac{\hbar e^{x+y}\left(2+\lambda-\sigma e^{x+y}\right) t^{\rho}}{\Gamma(\rho+1)}$, $\mu_{1}=-\frac{\hbar e^{x+y}\left(2-\mu+\sigma e^{x+y}\right) t^{\beta}}{\Gamma(\beta+1)}$, 


$$
\begin{aligned}
\xi_{2}= & \frac{\hbar(\hbar+1) e^{x+y}\left(2+\lambda-\sigma e^{x+y}\right) t^{\alpha} \rho}{\Gamma(\rho+1)} \\
& +\frac{\hbar^{2} e^{x+y}\left[\left(2+\lambda-\sigma e^{x+y}\right)\left(\lambda-\sigma e^{x+y}\right)+2\left(2+\lambda-4 \sigma e^{x+y}\right)\right] t^{2 \rho}}{\Gamma(2 \rho+1)} \\
& -\frac{\hbar^{2} \sigma e^{2(x+y)}\left(2-\mu+\sigma e^{x+y}\right) t^{\rho+\beta}}{\Gamma(\rho+\beta+1)}, \\
\eta_{2}=- & \frac{\hbar(\hbar+1) e^{x+y}\left(2-\mu+\sigma e^{x+y}\right) t^{\beta}}{\Gamma(\beta+1)} \\
& +\frac{\hbar^{2} e^{x+y}\left[\left(2-\mu+\sigma e^{x+y}\right)\left(\sigma e^{x+y}-\mu\right)+2\left(2-\mu+4 \sigma e^{x+y}\right)\right] t^{2 \beta}}{\Gamma(2 \beta+1)} \\
& +\frac{\hbar^{2} e^{2(x+y)}\left(2+\lambda-\sigma e^{x+y}\right) t^{\rho+\beta}}{\Gamma(\rho+\beta+1)},
\end{aligned}
$$

In this manner the remaining terms can be achieved.

The HATT solutions of the Eq. (1) having the initial condition (39) obtained in series form as $\xi=\xi_{0}+\xi_{1}+\xi_{2}+\cdots, \quad \eta=\eta_{0}+\eta_{1}+\eta_{2}+\cdots$

It's clear to see the Figs. 5-8 that the fractional predator-prey model exist and continuous with special variables. But prey population density firstly increase with special variables we choose here and then decrease. Although in the same way predator density always increases with these special variables, these behaviors accordingly the same obtained from HPM [35] which is the special case of HATT. We observed that the behavior of prey population density infected by parameter $\lambda$ and $\sigma$, but increasing rate of predator density infected by parameter $\sigma$ and $\mu$, which closed with realistic results observed in the field, with high precision at the third iteration. 


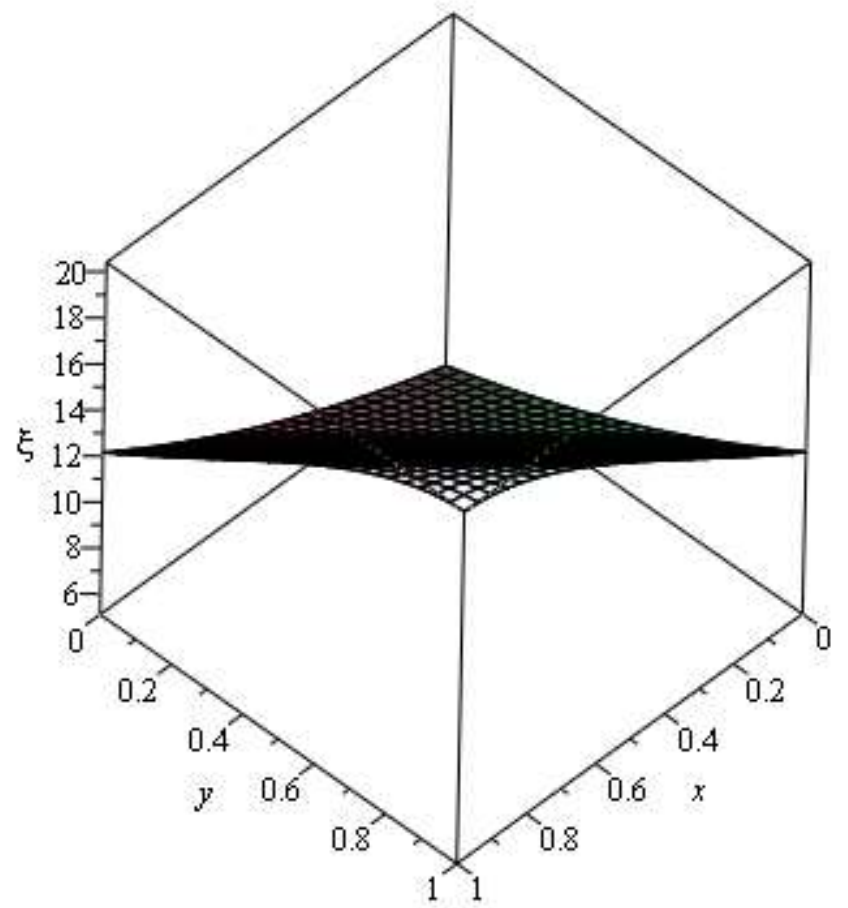

Figure 5. The shape presents the prey $\xi(x, y, t)$ population density with appropriate parameter for (41) when $\lambda=0.8, \sigma=0.04, \mu=0.3, \hbar=-1$ and $t=0.65$, with $\rho=\beta=1$. 


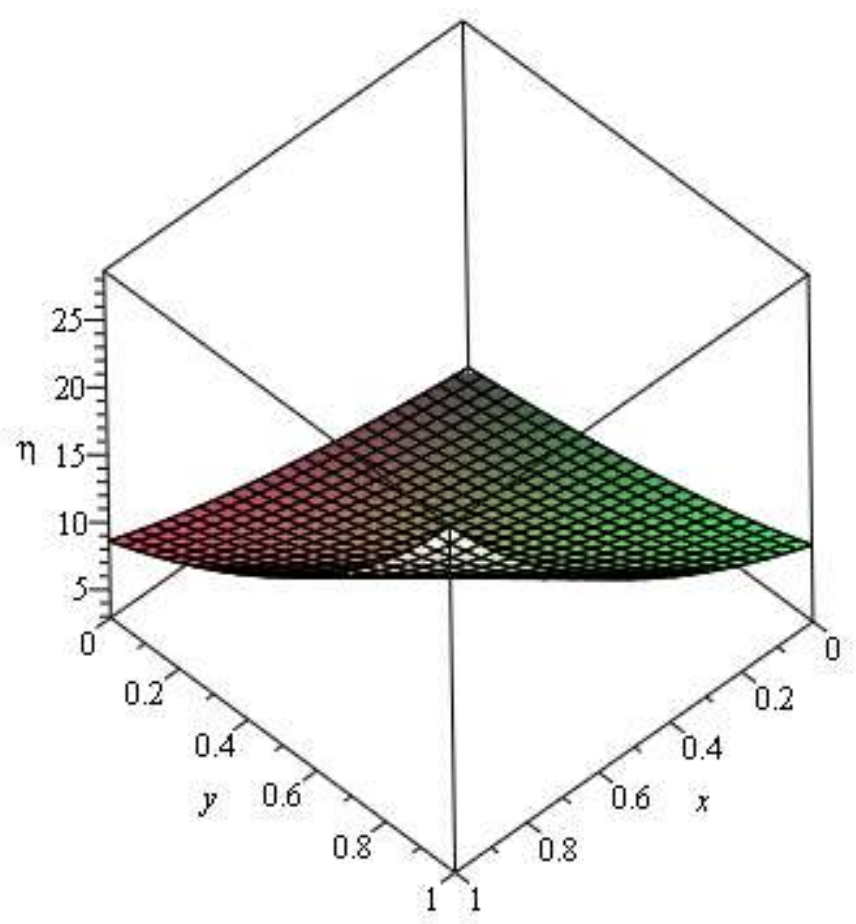

Figure 6. The shape presents predator $\eta(x, y, t)$ population density with appropriate parameter for (41) when $\lambda=0.8, \sigma=0.04, \mu=0.3, \hbar=-1$ and $t=0.65$, with $\rho=\beta=1$. 


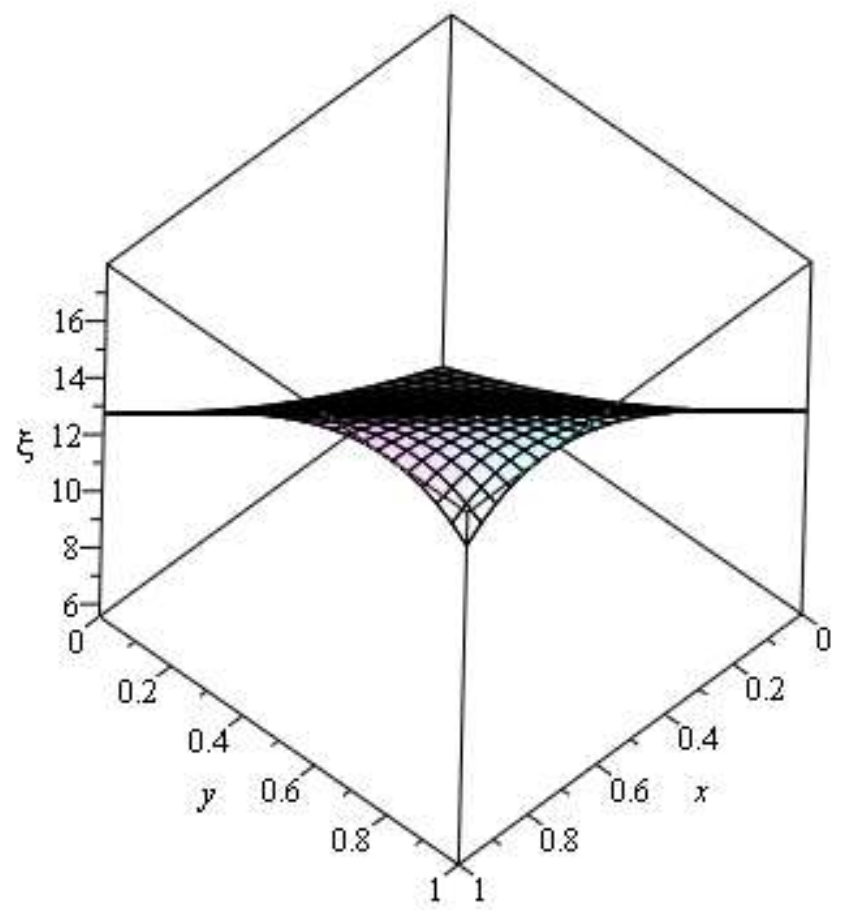

Figure 7. The shape presents the prey $\xi(x, y, t)$ population density with appropriate parameter for (41) when $\lambda=0.8, \sigma=0.04, \mu=0.3, \hbar=-1$ and $t=0.65$, with $\rho=0.95$ and $\beta=0.60$. 


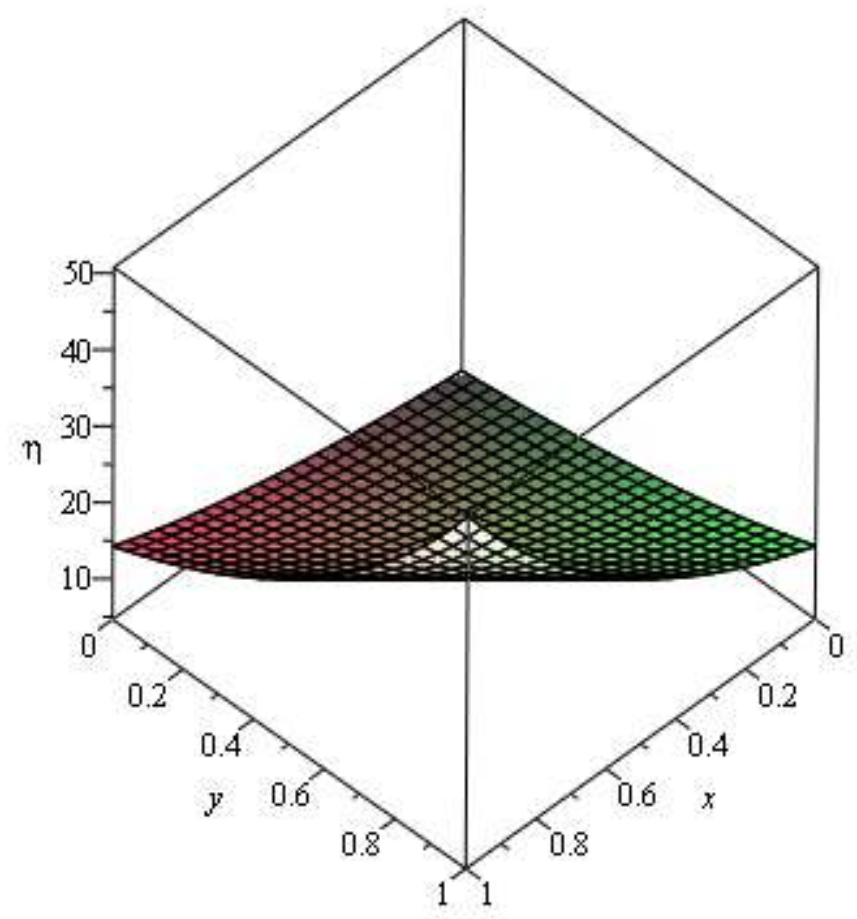

Figure 8. The shape presents predator $\eta(x, y, t)$ population density with appropriate parameter for (41) when $\lambda=0.8, \sigma=0.04, \mu=0.3, \hbar=-1$ and $t=0.65$, with $\alpha=0.95$ and $\beta=0.60$.

Case 3. Lastly, we take the predator-prey equation of fractional order having the initial conditions

$\xi(x, y, 0)=\sqrt{(x y),} \quad \eta(x, y, 0)=e^{x+y}$.

By using the HATT to solve the Eqs. (32) \& (33) with initial condition (42) we get the following results

$$
\begin{aligned}
& \xi_{0}=\sqrt{(x y)}, \quad \eta_{0}=e^{x+y}, \\
& \xi_{1}=\frac{\hbar\left(x^{2}+y^{2}-4 \lambda x^{2} y^{2}+4 a x^{2} y^{2} e^{x+y}\right) t^{\rho}}{4(x y)^{3 / 2} \Gamma(\rho+1)}, \quad \eta_{1}=-\frac{\hbar e^{x+y}(2-\mu+\sigma \sqrt{x y}) t^{\beta}}{\Gamma(\beta+1)}, \\
& \vdots \\
& \text { and so on, in this manner the remaining iterations can be achieved. }
\end{aligned}
$$


The HATT solutions of the Eq. (1) having the initial condition (42) obtained in series form is presented as

$\xi=\xi_{0}+\xi_{1}+\xi_{2}+\cdots, \quad \eta=\eta_{0}+\eta_{1}+\eta_{2}+\cdots$

We can observe from Figs. 9-12 that both population exit and increase with special variables and the behavior of prey population density infected by parameter $\lambda$ and $\sigma$, but increasing rate of predator density infected by parameter $\sigma$ and $\mu$, which closed with realistic results observed in the field, with accuracy at the second-term approximation. A higher number of iterations are needed to gain more approximation.

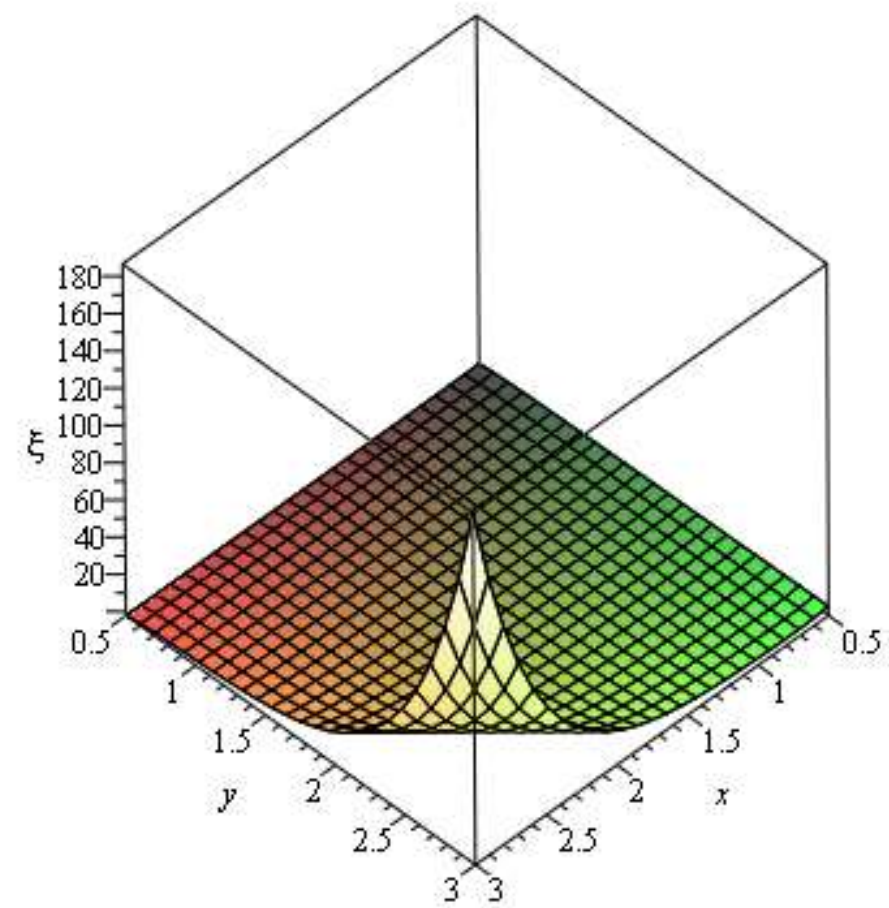

Figure 9. The shape presents the prey $\xi(x, y, t)$ population density with appropriate parameter for (44) when $\lambda=0.02, \sigma=0.06, \rho=0.3, \hbar=-1$ and $t=0.5$ with $\rho=\beta=1$. 


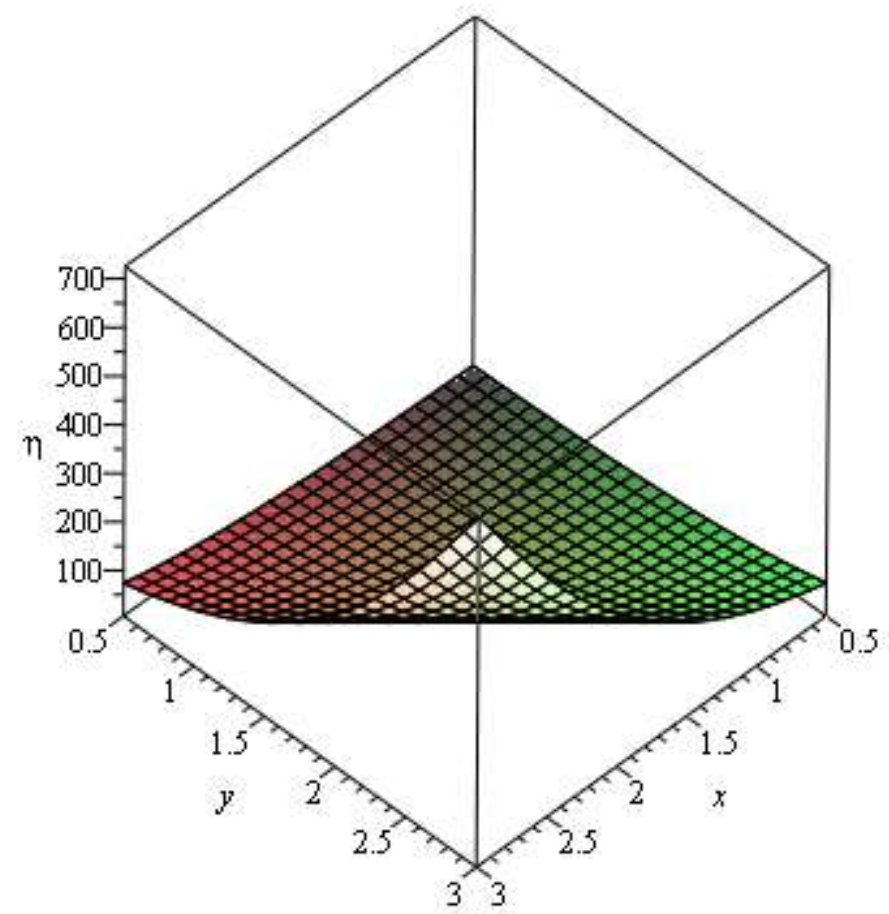

Figure 10. The shape presents predator $\eta(x, y, t)$ population density with appropriate parameter for (44) when $\lambda=0.02, \sigma=0.06, \mu=0.3, \hbar=-1$ and $t=0.5$, with $\rho=\beta=1$. 


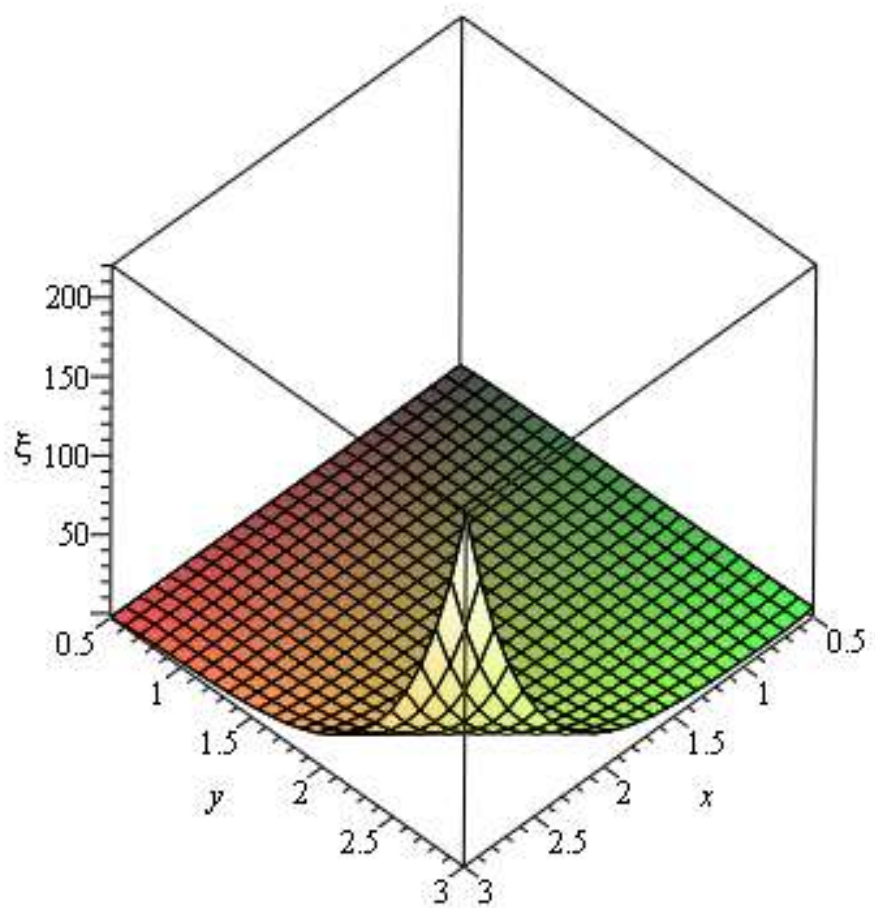

Figure 11. The shape presents the prey $\xi(x, y, t)$ population density with appropriate parameter for (44) when $\lambda=0.02, \sigma=0.06, \mu=0.3, \hbar=-1$ and $t=0.5$, with $\rho=0.95$ and $\beta=0.75$. 


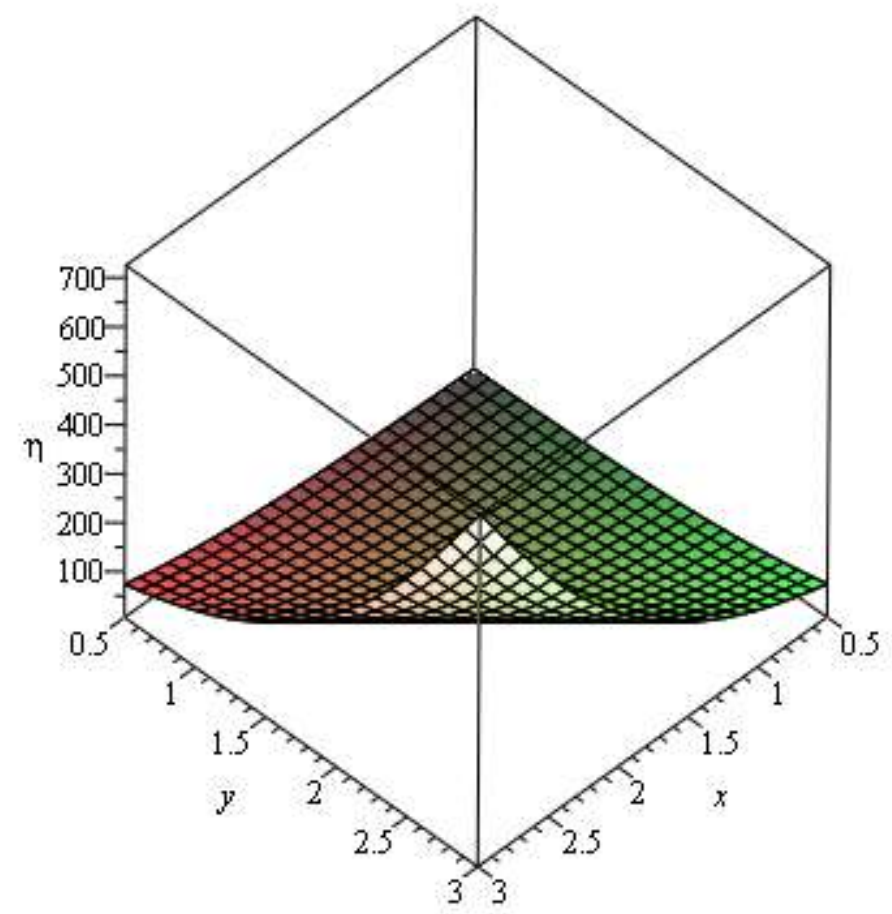

Figure 12. The shape presents predator $\eta(x, y, t)$ population density with appropriate parameter for (44) w when $\lambda=0.02, \sigma=0.06, \mu=0.3, \hbar=-1$ and $t=0.5$, with $\rho=0.95$ and $\beta=0.75$.

\section{Conclusions}

In this study, the homotopy analysis transform technique has been efficiently put up to find the approximate solution which converges to the exact solution of time-fractional nonlinear predator-prey population model subject to initial conditions. It's a significant outcome of these fractional ordered differential systems is that they gives higher degree of freedom and flexibility in the mathematical modelling and graphs show that the solution of these fractional systems is not only depends on time, but also depends on the order of fractional derivatives. The parameters $\rho$ and $\beta$ allow to control the speed of the motion of solutions close to the equilibrium points. The HATT displays a very fast convergence of the HATT solution and can yield uniformly valid 
approximation for both small and large parameters compared with standard perturbation method. The outcomes indicate that HATT a very strong and easy computational approach in obtaining analytical solution for various kinds of nonlinear fractional differential equations.

\section{References}

[1] K.B. Oldham and J. Spanier, The Fractional Calculus: Theory and application of differentiation and integration to arbitrary order (Dover Book on Mathematics), Academic Press, New York, NY, USA (1974), 234 pages.

[2] I. Podlubny, Fractional Differential Equations, Academic Press, San Diego, Calif, USA 198, (1999).

[3] S.G. Samko, A.A. Kilbas, O.L. Marichev, Frcational intergrals and derivatives: Theory and Applications, Gordon and Breach Science (1993).

[4] M. Rivero, J. Trujillo, L. Vazquez, M. Velasco, Fractional dynamics of populations, Applied Mathematics and Computation 218, (2011), 1089-1095.

[5] Y. K. Chang and J.J. Nieto, Some new existence results for fractional differential inclusions with boundary conditions, Math. Comput. Modelling 49, (2009), 605-609.

[6] A.M.A. El-Sayed, S.Z. Rida, A.A.M. Arafa, Exact solutions of fractional-order biological population model, Communications in Theoretical Physics 52, (2009), 992-996.

[7] A. Carpinteri and F. Mainardi, Fractional Calculus in Continuum Mechanics, Springer, New York, NY, USA (1997). 
[8] K.S. Miller and B. Ross, An Introduction to the fractional Calculus and fractional Differential equations, Willey, New York, NY, USA (1993).

[9] N.A. Khan, A. Ara, A. Mahmood, Approximate solution of time fractional chemical engineering equations: a comparative study, International Journal of Chemical Reactor Engineering A 19, (2010), 8 article.

[10] L. Song and H. Zhang, Application of homotopy analysis method to fractional KDV-BurgersKuramoto equation, Physics Letters A 367, (2007), 88-94.

[11] A. Abdon and E. Alabaraoye, Exact solutions fractional heat-like and wave-like equation with variable coefficients, Open Access Scientific Reports 2, (2013), doi.org/10.4172/Scientificreports, 633.

[12] M. Khan, M. A. Gondal, I. Hussain, S. Carimi Vanani, A new comparative study between homotopy analysis transform method and homotopy perturbation transform method on semi in finite domain, Mathematical and Computer Modeling 55, (2012), 1143-1150.

[13] D. Kumar, J. Singh and D. Baleanu, A fractional model of convective radial fins with temperature-dependent thermal conductivity, Romanian Reports in Physics, 69(1) (2017), 103

[14] J. Singh, M.M. Rashidi, Sushila, D. Kumar, A hybrid computational approach for JefferyHamel flow in non-parallel walls, Neural Computing and Applications, (2017), DOI: $10.1007 / \mathrm{s} 00521-017-3198-\mathrm{y}$

[15] D. Kumar, R. P. Agarwal and J. Singh, A modified numerical scheme and convergence analysis for fractional model of Lienard's equation, Journal of Computational and Applied Mathematics (2017), http://doi.org/10.1016/j.cam.2017.03.011 
[16] S. Abbasbandy, Homotopy analysis method for the Kawahara equation, Nonlinear Anal. Real World Application 11, (2010), 307-312.

[17] S. Nadeem, A. Hussain, M. Khan, Stagnation point of a Jeffrey fluid towards shrinking sheet, Zeitschrift fur Naturforschung 65, (2010), 540-548.

[18] S. J. Liao and K. F. Cheung, Homotopy analysis of nonlinear progressive waves in deep water, Journal of Engineering Mathematica 45, (2003), 105-116.

[19] L. Song and H. Zhang, Application of homotopy analysis method to fractional KDV-BurgersKuramoto equation, Physics Letters A 367, (2007), 88-94.

[20] W. Wu and S. J. Liao, Solving solitary waves with discontinuity by means of the homotopy analysis method, Chaos, Solitons and Fractals 26, (2005), 177-185.

[21] S.J. Liao, Beyond Perturbation: Introduction to the homotopy analysis method, Chaoman and Hall/CRC Press, Boca Raton (2003).

[22] A. Lotka, Element of physical biology, Williams and Wilkins, Baltimore (1925).

[23] V. Volterra, Variazioni e fluttuazioni del numero di individui in specie animali convivent, Mem. Accd. Linc. 2, (1926), 31-113.

[24] P.F. Verhulst, Notice sur la loi que la population suit dans son accrocisse-ment, Corr. Math. Phys. 10, (1838), 113-121.

[25] T.R. Malthus, An essay on the principle of population. Reprinted from 1798 edition, Johnson, London, as Malthus population: the first essay. Ann Arbor Paper-backs, University of Michigan, Ann Arbor Michigan, USA (1959). 
[26] H.I. Freedman, Deterministic mathematical model in population ecology, Marcel Dekker, New York 57, (1980), 95-252.

[27] S.R. Dunbar, Travelling wave solutions of diffusive Lotka-Volterra equation, Journal of Mathematical Biology 17, (1983), 11-32.

[28] R. Ardit and R. Ginzburg, Coupling in predator-prey dynamics: ratio-dependence, J. Theor. Biol. 139, (1989), 311-326.

[29] S.A. Gourely and N.F. Britton, Apredator-prey reaction-diffusion system with nonlocal effects, Journal of Mathematical Biology 34, (1996), 297-333.

[30] R. Devaney, M. Hirsch, S. Smale, Differential equations, dynamical systems and an interoduction to Chaos (2da. Ed), USA: Academic Press (2004).

[31] S. Petrovskii, H. Malchow, B.L. Li, An exact solution of a diffusive predator-prey system, Proceedings of The Royal Society of London A 161, (2005), 1029-1053.

[32] H. Boudjellaba and T. Sari, Dynamic transcritical bifurcations in a class of slow-fast predatorprey models, J. Diff. Eq. 246, (2009), 2205-2225.

[33] E. Ahmed, A.M.A. El-Sayed, H.A.A. El-Saka, Equilibrium points, stability and numerical solutions of fractional order predator-prey and rabies models, J. Math. Anal. Appl. 325, (2007), 542-553.

[34] S. Das, P.K. Gupta, Rajeev, A fractional predator prey model and its solution, Int. J. Nonlin. Sci. Numer. Simul. 10, (2009), 873-876. 
[35] L. Yanqin and X. Baogui, Numerical solutions of a fractional predator-prey system, Advances Difference Equations 2011, (2011), 11 pages.

[36] M. Caputo, Elasticita e Dissipazione, Zani-Chelli, Bologna (1969).

[37] A.A. Kilbas, H.M. Srivastava, J.J. Trujillo, Theory and Applications of Fractional Differential Equations, Elsevier Science B.V., Amsterdam 204, (2006). 